\title{
A Successful Pregnancy in a Patient with Chronic Myeloid Leukemia Exposed to Nilotinib during the Entire First Trimester of Pregnancy
}

\author{
Cili .A, Ivanaj .A \\ Hematology clinic, University Hospital Center “Mother Teresa”, Tirana, Albania
}

\begin{abstract}
The occurrence of pregnancy in chronic myeloid leukemia in pregnancy is rare and its management poses a clinical challenge for physicians treating these patients. In February 2010 during a routine examination, a 25-year-old woman was diagnosed with Philadelphia-positive CML in chronic phase. She was normal upon physical examination without clinical symptoms. Laboratory studies showed hemoglobin $10.7 \mathrm{~g} / \mathrm{dL}$, platelets $101 \times 10^{9} \mathrm{~L}$ and white cell count $54.8 \times 10^{9} \mathrm{~L}$ with a differential revealing $7 \%$ myelocytes, $2 \%$ promyelocytes, $15 \%$ metamyelocytes, $28 \%$ band cells, $39 \%$ neutrophils, $3 \%$ eosinophils, $0 \%$ basophils, $4 \%$ lymphocytes and $2 \%$ monocytes. The diagnosis of CML was confirmed based on bcr-abl mRNA transcript detection and conventional chromosome banding, which revealed a 46, XY, t(9;22) karyotype. The patient started treatment with Imatinib $100 \mathrm{mg}, 4$ tabs po/d. In March 2013 the patient under Imatinib treatment lost MMR so we started treatment with Nilotinib $300 \mathrm{mg} / \mathrm{po} / \mathrm{d}$. In December 2013, the patient was in complete cytogenetic remission and major molecular response. In January 2014 the patient came for a routine visit in our clinic and she revealed she became pregnant while she was on nilotinib. The pregnancy was identified at 13weeks. Because the patient elected to continue her pregnancy, nilotinib was stopped immediately, and no further treatment was given until delivery. Neither obstetrical complications nor a structural malformation in neonates was observed. The baby's growth and development have been normal. Although this experience is limited to a single patient, the success of this patient demonstrates that the management of chronic myeloid leukemia in pregnant women may be individualized based on the relative risks and benefits of the patient and fetus.
\end{abstract}

Keyword: CML, teratogenic, pregnant, fetus, nilotinib

\section{Introduction}

Chronic myelogenous leukemia (CML) is a myeloproliferative disorder characterized by increased proliferation of the granulocytic cell line without the loss of their capacity to differentiate. Consequently, the peripheral blood cell profile shows an increased number of granulocytes and their immature precursors, including occasional blast cells. CML is an acquired abnormality that involves the hematopoietic stem cell. It is characterized by a cytogenetic aberration consisting of a reciprocal translocation between the long arms of chromosomes 22 and $9[\mathrm{t}(9 ; 22)]$. The translocation results in a shortened chromosome 22, an observation first described by Nowell and Hungerford and subsequently termed the Philadelphia (Ph1) chromosome after the city of discovery. This translocation relocates an oncogene called $\mathrm{ABL}$ from the long arm of chromosome 9 to a specific breakpoint cluster region (BCR) in the long arm of chromosome 22. The ABL oncogene encodes a tyrosine protein kinase. The resulting $\mathrm{BCR} / \mathrm{ABL}$ fusion gene encodes a chimeric protein with strong tyrosine kinase activity.

The introduction of the TK inhibitor (TKI) imatinib in 1998 indisputably advanced the clinical management of cancer. Imatinib has demonstrated its efficacy by increasing overall survival and substantially improving the life expectancy and quality of life of patients with CML. However, the occurrence of CML during pregnancy poses a unique clinical challenge for physicians treating these patients and requires balancing concerns between maternal survival and fetal health in both the short- and long-term. Because imatinib was teratogenic in rats, it was strongly advised that effective contraception be used during therapy to prevent pregnancy. The inability of patients to tolerate treatment and the emergence of bcr-abl mutations that reduced the binding affinity of imatinib prompted pharmaceutical research that led to the discovery of similarly effective, targeted, second generation TKIs such as nilotinib and dasatinib. There is still insufficient efficacy and safety data on these newer medications to warrant their safety in pregnant women with CML. In this study, we are reporting the outcome of a patient with CML who became pregnant under nilotinib treatment. The patient revealed she was pregnant only at 13 weeks of pregnancy and she refused interrupting the pregnancy.

\section{Case Description}

In February 2010 during a routine examination, a 25-yearold woman was diagnosed with Philadelphia-positive CML in chronic phase. She was normal upon physical examination without clinical symptoms. Laboratory studies showed hemoglobin $10.7 \mathrm{~g} / \mathrm{dL}$, platelets $101 \times 10^{9}$ $\mathrm{L}$ and white cell count $54.8 \times 10^{9} \mathrm{~L}$ with a differential revealing $7 \%$ myelocytes, $2 \%$ promyelocytes, $15 \%$ metamyelocytes, $28 \%$ band cells, 39\% neutrophils, 3\% eosinophils, $0 \%$ basophils, 4\% lymphocytes and $2 \%$ monocytes. The diagnosis of CML was confirmed based on bcr-abl mRNA transcript detection and conventional chromosome banding, which revealed a 46, XY, t(9;22) karyotype. The patient started treatment with Imatinib 400 mg po/d. In March 2013 the patient under Imatinib treatment lost MMR so we started treatment with Nilotinib $300 \mathrm{mg} / \mathrm{po} / \mathrm{d}$.

In December 2013, the patient was in complete cytogenetic remission and major molecular response. In January 2014 the patient came for a routine visit in our 


\section{International Journal of Science and Research (IJSR) \\ ISSN (Online): 2319-7064}

Index Copernicus Value (2013): 6.14 | Impact Factor (2014): 5.611

clinic and she revealed she became pregnant while she was on nilotinib. The pregnancy was identified at 13weeks. The patient was informed of the potential fetal toxicities of therapy. After detailed and meticulous counseling, the patient elected to continue her pregnancy, so nilotinib was stopped immediately, and no further treatment was given until delivery. A follow-up with ultrasound scans during the course of the pregnancy was unremarkable. In July 2014, she delivered via cesarean section a healthy male baby weighing $3.8 \mathrm{~kg}$. The baby was never breastfed. At 6 months post-partum, the patient's child has been healthy and developing normally. After delivery, the patient lost molecular, cytogenetic and HR. The patient was put on Nilotinib treatment again with the same dosage. She is currently in MMR.

\section{Discussion}

CML comprises less than $10 \%$ of leukemias in pregnancy and is very rare during conception; the incidence has been estimated as 1 per 100, 000 pregnancies annually. Over the last few decades, treatment of CML in pregnancy has consisted of conservative management with varying degrees of success including leukapheresis, hydroxyurea, and interferon. The therapeutic management of CML in pregnant women with targeted therapies often presents divisive dilemmas and poses substantial challenges to both patients and their physicians. The key question to consider is whether to stay on these agents, which carry the risks of birth defects, or stop the medications and risk relapse. Although most of the existing data on the effects of imatinib on pregnancy have shown satisfactory outcomes, they do not indicate that it can be safely recommended during the first trimester of gestation. One of the most comprehensive data sets on the effect of imatinib on pregnancy was recently reported by Pye and colleagues. In Pye et al.'s study, imatinib was evaluated in 180 women who were exposed to treatment during pregnancy; outcomes were available for 125 patients. In total, 50\% delivered a healthy baby, $28 \%$ elected to have a termination and $14 \%$ had a miscarriage. Twelve pregnancies resulted in infants with fetal abnormalities; 3 of which had strikingly similar complex malformations. In regards to the second generation TKIs, a literature search revealed only one report on the use of dasatinib during pregnancy. Nilotinib, a potent TKI, was introduced in November 2007 for the treatment of patients with chronic or acute phase CML who were resistant to or intolerant of imatinib. Evidence from an in-vitro study indicated that nilotinib was 20 times more potent than imatinib against cells expressing the wild-type bcr-abl. Compared to imatinib, nilotinib also has a higher binding affinity and selectivity for the inactive abl kinase conformation. Currently, nilotinib is classified as US Food and Drug Administration Pregnancy Category D with studies in rabbits showing it is associated with mortality, abortion and decreased gestational weights at a dose of 300 $\mathrm{mg} / \mathrm{kg} / \mathrm{d}$ (approximately half of the exposure used in humans based on area-under-curve (AUC)) with an overall lack of data in humans. Based on the medical information provided by the manufacturer, nilotinib is not considered teratogenic, but an increased risk of embryo toxicity was noted at even sub-therapeutic doses. Similar to other TKIs, the manufacturer states that nilotinib is contraindicated during conception because of concerns it may cause fetal deformities. However, the medication safety information of nilotinib in pregnancy is obtained through animal studies, which may not apply to humans. A review of the literature did not identify any published data in women with CML who were treated with nilotinib when they conceived. In our patient, the first fetus has been exposed to therapy throughout the first trimester of pregnancy during embryogenesis (weeks 8 to 12 of gestation) and 1 week after. We found no published studies on the use of nilotinib during pregnancy, apart from the case report described above. In our case, pregnancy progressed normally and the infant was delivered at term without complications. There was no congenital anomalies and no late adverse effect, the baby being now 15 months old.

Although nilotinib treatment did not have a negative impact on this patient and her fetus, patients receiving nilotinib should be advised to practice adequate contraception. If the patient becomes pregnant while receiving the drug, the patient should be advised of the potential hazard to the fetus, and the drug should be discontinued.

\section{Conclusion}

Although this experience is limited to a single patient, the success of this patient demonstrates that the management of CML in pregnant women may be individualized based on the relative risks and benefits of the patient and fetus and that the patient must be involved in decisions.

\section{References}

[1] Rowley JD. Letter: A new consistent chromosomal abnormality in chronic myelogenous leukaemia identified by quinacrine fluorescence and Giemsa staining. Nature. 1973; 243:290-3. doi: 10.1038/243290a0.[PubMed] [Cross Ref]

[2] Hensley ML, Ford JM. Imatinib treatment: specific issues related to safety, fertility, and pregnancy. Semin Hematol. 2003; 40:21-5. [PubMed]

[3] Lichtman M, L J. Acute myelogenous leukemia. In: Beutler E, Lichtman M, Coller B, et al, editor. Williams Hematology. 6. New York, NY, McGrawHill; 2001. p. 1047.

[4] Bazarbashi MS, Smith MR, Karanes C, Zielinski I, Bishop CR. Successful management of $\mathrm{Ph}$ chromosome chronic myelogenous leukemia with leukapheresis during pregnancy. Am J Hematol. 1991; 38:235-7. doi: 10.1002/ajh.2830380316. [PubMed] [Cross Ref]

[5] Strobl FJ, Voelkerding KV, Smith EP. Management of chronic myeloid leukemia during pregnancy with leukapheresis. J Clin Apher. 1999; 14:42-4. doi: 10.1002/(SICI)1098-1101(1999)14:1<42::AIDJCA8>3.0.CO;2-2. [PubMed] [Cross Ref]

[6] Celiloglu M, Altunyurt S, Undar B. Hydroxyurea treatment for chronic myeloid leukemia during pregnancy. Acta Obstet Gynecol Scand. 2000;79:8034. doi: 10.3109/00016340009169201. [PubMed] [Cross Ref]

\section{Volume 4 Issue 11, November 2015}




\section{International Journal of Science and Research (IJSR) \\ ISSN (Online): 2319-7064}

Index Copernicus Value (2013): 6.14 | Impact Factor (2014): 5.611

[7] Patel M, Dukes IA, Hull JC. Use of hydroxyurea in chronic myeloid leukemia during pregnancy: a case report. Am J Obstet Gynecol. 1991; 165:565-6. [PubMed]

[8] Baer MR, Ozer H, Foon KA. Interferon-alpha therapy during pregnancy in chronic myelogenous leukaemia and hairy cell leukaemia. Br J Haematol. 1992; 81:167-9. doi: 10.1111/j.13652141.1992.tb08202.x.[PubMed] [Cross Ref]

[9] Baykal C, Zengin N, Coskun F, Guler N, Ayhan A. Use of hydroxyurea and alpha-interferon in chronic myeloid leukemia during pregnancy: a case report. Eur J Gynaecol Oncol. 2000; 21:89-90. [PubMed]

[10] Kuroiwa M, Gondo H, Ashida K, Kamimura T, Miyamoto T, Niho Y, Tsukimori K, Nakano H, Ohga $\mathrm{S}$. Interferon-alpha therapy for chronic myelogenous leukemia during pregnancy. Am J Hematol.1998; 59:101-2. doi: 10.1002/(SICI)10968652(199809)59:1<101::AID-AJH23>3.0.CO;2-

D. [PubMed] [Cross Ref]

[11] AlKindi S, Dennison D, Pathare A. Imatinib in pregnancy. Eur J Haematol. 2005; 74:535-7. doi: 10.1111/j.1600-0609.2005.00420.x. [PubMed] [Cross Ref]

[12] Prabhash K, Sastry PS, Biswas G, Bakshi A, Prasad N, Menon H, Parikh PM. Pregnancy outcome of two patients treated with imatinib. Ann Oncol. 2005; 16:1983-4. doi: 10.1093/annonc/mdi398. [PubMed] [Cross Ref]

[13] Choudhary DR, Mishra P, Kumar R, Mahapatra M, Choudhry VP. Pregnancy on imatinib: fatal outcome with meningocele. Ann Oncol. 2006; 17:178-9. doi: 10.1093/annonc/mdj065. [PubMed] [Cross Ref]

[14] Ault P, Kantarjian H, O'Brien S, Faderl S, Beran M, Rios MB, Koller C, Giles F, Keating M, Talpaz M, et al. Pregnancy among patients with chronic myeloid leukemia treated with imatinib. J Clin Oncol.2006; 24:1204-8. doi: 10.1200/JCO.2005.04.6557. [PubMed] [Cross Ref]

[15] Pye SM, Cortes J, Ault P, Hatfield A, Kantarjian H, Pilot R, Rosti G, Apperley JF. The effects of imatinib on pregnancy outcome. Blood. 2008; 111:5505-8. doi: 10.1182/blood-2007-10-114900. [PubMed][Cross $\underline{\text { Ref] }}$

[16] Cortes J, O'Brien S, Ault P, Borthakur G, Jabbour E, Bradley-Garelik B, Debreczeni K, Yang D, Liu D, Kantarjian H. Pregnancy Outcomes among Patients with Chronic Myeloid Leukemia Treated with Dasatinib. Blood. 2008. (ASH Annual Meeting Abstracts):112: (Abstract 3230)

[17] O'Hare T, Walters DK, Stoffregen EP, Jia T, Manley PW, Mestan J, Cowan-Jacob SW, Lee FY, Heinrich $\mathrm{MC}$, Deininger MW, et al. In vitro activity of Bcr-Abl inhibitors AMN107 and BMS-354825 against clinically relevant imatinib-resistant $\mathrm{Abl}$ kinase domain mutants. Cancer Res. 2005; 65:4500-5. doi: 10.1158/0008-5472.CAN-05-0259. [PubMed] [Cross $\underline{\text { Ref] }}$ 